\title{
CFO Estimation for OFDM-based Massive MIMO Systems in Asymptotic Regime
}

\author{
Parna Sabeti ${ }^{\star}$, Arman Farhang ${ }^{\dagger}$, Nicola Marchetti ${ }^{\star}$ and Linda Doyle ${ }^{\star}$ \\ * CONNECT, Trinity College Dublin, Ireland \\ $\dagger$ CONNECT, University College Dublin, Ireland \\ Email: sabetip@tcd.ie, arman.farhang@ucd.ie, \{nicola.marchetti, ledoyle\}@tcd.ie
}

\begin{abstract}
Massive multiple input multiple output (MIMO) plays a pivotal role in the fifth generation $(5 \mathrm{G})$ wireless networks. However, the carrier frequency offset (CFO) estimation is a challenging issue in the uplink of multi-user massive MIMO systems. In fact, frequency synchronization can impose a considerable amount of computational complexity to the base station (BS) due to a large number of BS antennas. In this paper, thanks to the properties of massive MIMO in the asymptotic regime, we develop a simple synchronization technique and derive a closed form equation for CFO estimation. We show that the phase information of the covariance matrix of the received signals is solely dependent on the users' CFOs. Hence, if a real-valued pilot is chosen, the CFO values can be straightforwardly calculated from this matrix. Hence, there is no need to deal with a complex optimization problem like the other existing CFO estimation techniques in the literature. Our simulation results testify the efficacy of our proposed CFO estimation technique. As we have shown, the performance of our method does not deteriorate as the number of users increases.
\end{abstract}

\section{INTRODUCTION}

Massive multiple input multiple output (MIMO) is one of the key technologies for the fifth generation $(5 \mathrm{G})$ wireless networks, [1], [2]. It can significantly improve network capacity by enabling users to simultaneously utilize the entire available bandwidth. [3], [4]. Moreover, the emergence of millimeter wave (mmWave) systems, where large antenna arrays play a crucial role, has highlighted the importance of massive MIMO even further, [5].

Apart from all the advantages, massive MIMO systems tremendously suffer from synchronization errors, such as, timing offset (TO) and carrier frequency offset (CFO), [6], [7]. In orthogonal frequency division multiplexing (OFDM), the effects of TO can be absorbed into a cyclic prefix (CP) provided an adequately long guard interval is used, [8]. However, CFO adversely affects the performance of OFDM and is a challenging problem especially in the presence of multiple-users with different CFOs. Although, there has been substantial amount of work reported on the frequency synchronization for conventional multi-user OFDM systems, [9]-[11], not all of them are applicable to massive MIMO systems.

In the past decade, a number of studies have been conducted to address the synchronization problem in MIMO OFDM systems. The authors in [12] developed an algorithm for joint CFO compensation and multi-user detection for small-scale multi-user MIMO systems. However, computational complexity of such an approach becomes prohibitive for large scale antenna systems (LSAS). The authors of [13] proposed a joint
CFO and channel estimation with maximum likelihood (ML) estimator. This algorithm is very complex as it requires a multidimensional grid search. Later, the authors in [14], converted the ML CFO estimator into a set of line search problems. However, this algorithm requires per antenna CFO estimation for all the users. This imposes a great amount of complexity to the receiver. In [15], a two stage pilot-based CFO and phase noise (PN) compensation for point to point high frequency MIMO OFDM was proposed. Both stages of this algorithm are based on the least squares (LS) criterion. However, solution of these LS-based problems involves complex matrix inversion operations. This leads to a substantial amount of complexity in massive MIMO systems.

The authors in [16], considered a single-carrier massive MIMO system, and proposed impulse-like pilot sequences to provide time-domain orthogonality among different users. Then, the estimated CFOs need to be fed back to multiple users over an error free control channel, so that, the users can align their local oscillators. Moreover, the approach in [16] requires a long pilot sequence for an accurate estimation. More recently, the authors in [17] and [18] proposed a spatially averaged periodogram based technique with a constant envelope pilot. However, this technique still suffers from the need for long training sequences. The authors in [19], proposed a frequency synchronization technique for massive MIMO OFDM by exploiting the angle of arrival information of the users. However, when the users are not spatially separated, their angles of arrival might be about the same. As a result, the users' signals cannot be accurately distinguished from each other. In [20], an improved user grouping scheme has been designed to deal with this issue, where the CFO estimation and data detection are jointly performed for the users that are close to one another. Despite the imposed computational complexity due to the multi-user interference (MUI) cancellation, this technique still cannot accurately compensate the CFO effects. This becomes very challenging in dense networks, where many users are located very close to each other.

In this paper, we propose a simple CFO estimation method for the uplink of OFDM-based multi-user massive MIMO systems. We show that if we multiply the received signal at each BS antenna to its Hermitian, and average the result over all BS antennas, this matrix tends to the covariance matrix of the received signals in the asymptotic regime. Then, we show that the phase information of this matrix solely depends on the users' CFOs. Hence, provided that real-valued pilots are 
used, the angles of the elements of this matrix are a linear combination of the users' CFOs. Therefore, unlike other existing CFO estimation techniques, we do not need to solve any optimization problem and the CFOs can be directly estimated. We derive a closed form equation for estimation of different users' CFOs. Furthermore, numerical results are presented to verify the performance of our proposed synchronization technique. The full-range CFO, i.e. $[-0.5,0.5)$, is considered in our simulation. We demonstrate that one OFDM symbol is sufficient as a pilot for CFO estimation. Thus, we do not need a long pilot for CFO estimation. We also show that the performance of our proposed technique does not suffer from MUI as the number of users increases. Moreover, the performance of our method can be enhanced by increasing the number of BS antennas.

The rest of the paper is organized as follows. Section II presents the system model for the uplink of OFDM-based multi-user massive MIMO system in presence of CFOs. In Section III, we present our proposed CFO estimation technique. CFO compensation is briefly explained in Section IV. Numerical analysis is provided in Section V. Finally, the conclusions are drawn in Section VI.

Notation: Matrices, vectors and scalar quantities are denoted by boldface uppercase, boldface lowercase and normal letters, respectively. The $i^{\text {th }}$ element of the vector a and the $(i, j)^{\text {th }}$ element of the matrix $\mathbf{A}$ are denoted as $a[i]$ and $A[i, j]$, respectively. Superscripts $(.)^{\mathrm{H}},(.)^{\mathrm{T}}$ and $(.)^{-1}$ denote Hermitian, transpose and the inverse of a matrix, respectively. $\mathbf{I}_{N}$ is an $N \times N$ identity matrix, $\mathbb{E}\{$.$\} denotes the expectation operator,$ and symbol $\otimes$ stands for circular convolution operation. $\angle a$ indicates the angle of the complex number $a . \mathbf{d}=\operatorname{diag}(\mathbf{D})$ is the vector including the elements on the main diagonal of the matrix $\mathbf{D}$, and $\mathbf{C}=\operatorname{circ}(\mathbf{c})$ is a circulant matrix with the first column c. Finally, $\operatorname{Rect}_{b}[i-a]$ is a rectangular pulse centered at $a$ and with the width $b$.

\section{SySTEM MODEL}

Consider the uplink of an OFDM-based massive MIMO system where $P$ single antenna users are communicating with a BS equipped with $M \gg P$ antennas. We assume that different users' wireless channels are statistically independent and time invariant during one OFDM symbol. Hence, they can simultaneously share all the available subcarriers as their signals can be distinguished through their respective channel gains. Having $N$ active subcarriers, the $N \times 1$ vector of the $\kappa^{\text {th }}$ OFDM symbol for user $p$ can be obtained as

$$
\mathbf{x}_{p}^{\kappa}=\mathbf{F}_{N}^{\mathrm{H}} \mathbf{d}_{p}^{\kappa},
$$

where the $N \times 1$ vector $\mathbf{d}_{p}^{\kappa}$ contains the $\kappa^{\text {th }}$ data symbol of user $p$, which is normalized to have a power of unity. Also, $\mathbf{F}_{N}$ is the normalized $N$-point DFT matrix with the elements $F_{N}[i, k]=\frac{1}{\sqrt{N}} e^{-j \frac{2 \pi}{N} i k}$ for $i, k=0, \cdots, N-1$. The CP length, $N_{\mathrm{CP}}$, is considered to be longer than the channel impulse response (CIR) length, $L$, and the largest user's TO in order to have a perfect time synchronization. In addition, we assume a collocated BS model where the BS is equipped with coherent oscillators at all the antennas. Thus, the amount of each user's CFO is the same for all the BS antennas. After CP removal, the time domain representation of an OFDM symbol received at the $m^{\text {th }}$ BS antenna can be written as

$$
\mathbf{r}_{m}^{\kappa}=\sum_{p=0}^{P-1} \boldsymbol{\Phi}_{p}^{\kappa} \mathbf{X}_{p}^{\kappa} \mathbf{h}_{m, p}+\mathbf{n}_{m},
$$

where $\mathbf{X}_{p}^{\kappa}$ is an $N \times L$ matrix including the first $L$ columns of the circulant matrix $\operatorname{circ}\left(\mathbf{x}_{p}^{\kappa}\right), \mathbf{n}_{m} \sim \mathcal{C N}\left(0, \sigma_{n}^{2} \mathbf{I}_{N}\right)$ is the complex additive white Gaussian noise (AWGN) with the variance of $\sigma_{n}^{2}$ at the $m^{\text {th }}$ antenna and $\mathbf{h}_{m, p}$ is the $L \times 1$ CIR vector between user $p$ and the $m^{\text {th }} \mathrm{BS}$ antenna. We assume the channel taps to be a set of independent and identically distributed (i.i.d.) random variables that follow a complex normal distribution $\mathcal{C N}\left(0, \boldsymbol{\rho}_{p}\right)$, where $\boldsymbol{\rho}_{p}$ is an $L \times 1$ vector of the $p^{\text {th }}$ user's channel power delay profile (PDP). Also, $\boldsymbol{\Phi}_{p}^{\kappa}$ is an $N \times N$ diagonal CFO matrix with the diagonal elements $\Phi_{p}^{\kappa}[l, l]=e^{j \frac{2 \pi}{N} \epsilon_{p}\left(l+\left(N+N_{\mathrm{CP}}\right) \kappa+N_{\mathrm{CP}}\right)}$ for $l=0, \cdots, N-1$, and $\epsilon_{p}$ is the CFO normalized to subcarrier spacing.

\section{CFO ESTIMATION}

In this section, we propose a pilot-based CFO estimation technique for the introduced system model. We consider the first OFDM symbol within each data packet as users' pilots. To keep the formulation simple, without any loss of generality, we omit the symbol index in our derivation, i.e. the superscript $\kappa$. If we multiply the first received OFDM symbol at each antenna to its Hermitian and average them over all the BS antennas, we have

$$
\begin{aligned}
\mathbf{R} & =\frac{1}{M} \sum_{m=0}^{M-1} \mathbf{r}_{m} \mathbf{r}_{m}^{\mathrm{H}} \\
& =\sum_{p=0}^{P-1} \sum_{q=0}^{P-1} \boldsymbol{\Lambda}_{p} \mathbf{C}_{p, q} \boldsymbol{\Lambda}_{q}^{\mathrm{H}}+\frac{1}{M} \sum_{m=0}^{M-1} \mathbf{n}_{m} \mathbf{n}_{m}^{\mathrm{H}} \\
& +\frac{1}{M} \sum_{m=0}^{M-1}\left(\sum_{p=0}^{P-1} \boldsymbol{\Lambda}_{p} \mathbf{h}_{m, p}\right) \mathbf{n}_{m}^{\mathrm{H}}+\frac{1}{M} \sum_{m=0}^{M-1} \mathbf{n}_{m}\left(\sum_{q=0}^{P-1} \boldsymbol{\Lambda}_{q} \mathbf{h}_{m, q}\right)^{\mathrm{H}},
\end{aligned}
$$

where $\boldsymbol{\Lambda}_{p}=\boldsymbol{\Phi}_{p} \mathbf{X}_{p}$ and $\mathbf{C}_{p, q} \triangleq \frac{1}{M} \sum_{m=0}^{M-1} \mathbf{h}_{m, p} \mathbf{h}_{m, q}^{\mathrm{H}}$. In the asymptotic regime, i.e. $M \rightarrow \infty$, the two last terms in (3) tend to zero and the second one becomes a diagonal matrix equal to $\sigma_{n}^{2} \mathbf{I}_{N}$. Hence, for large values of $M, \mathbf{R}$ can be written as

$$
\mathbf{R}=\sum_{p=0}^{P-1} \sum_{q=0}^{P-1} \boldsymbol{\Lambda}_{p} \mathbf{C}_{p, q} \boldsymbol{\Lambda}_{q}^{\mathrm{H}}+\sigma_{n}^{2} \mathbf{I}_{N} .
$$

Moreover, according to the law of large numbers, as $M$ grows large, $C_{p, q}[i, j] \rightarrow \mathbb{E}\left\{h_{m, p}[i] h_{m, q}^{*}[j]\right\}$. Since $h_{m, p}[i]$ is a set of i.i.d. random variables, $\mathbb{E}\left\{h_{m, q}[i] h_{m, q}^{*}[j]\right\}=0$ for $i \neq j$ and $p \neq q$. Thus, $\mathbf{C}_{p, q}$ is a zero matrix for $p \neq q$, and $\mathbf{C}_{p, p}$ 
is a real-valued diagonal matrix with the main diagonal $\boldsymbol{\rho}_{p}$. Therefore, based on equation (4), each entry of $\mathbf{R}$ is given by

$$
\begin{aligned}
R[i, j] & =\sum_{p=0}^{P-1} \Phi_{p}[i, i] \sum_{l=0}^{N-1} X_{p}[i, l] C_{p, p}[l, l] X_{p}^{\mathrm{H}}[l, j] \Phi_{p}^{\mathrm{H}}[j, j] \\
& =\sum_{p=0}^{P-1} e^{j \frac{2 \pi}{N} \epsilon_{p}(i-j)} \sum_{l=0}^{N-1} X_{p}[i, l] C_{p, p}[l, l] X_{p}^{\mathrm{H}}[l, j] .
\end{aligned}
$$

Accordingly, if we choose $\mathbf{X}_{p}$ to be real, the angles of the elements of matrix $\mathbf{R}$ will be a linear function of the users' CFOs. Note that due to the summation over $p$ in equation (5), in order to be able to estimate different users' CFOs, we need to choose orthogonal pilot sequences for different users in the time domain. To this end, a pilot should take zero value where other users' pilots are non-zero. In addition, due to the channel delay spread, users' pilots spread over $L$ samples. Thus, an additional guard of $L$ zero samples is required between users' pilot elements. Considering these two points, we propose a pilot signal that has $L$ non-zero elements every $2 L P$ samples in the time-domain, which can be given by $\mathbf{d}_{p}=\mathbf{F}_{N} \gamma_{p}$ in the frequency domain where $\gamma_{p}$ is an $N \times 1$ vector. The elements of $\gamma_{p}$ can be shown as

$$
\left.\gamma_{p}[i]=\sum_{a=0}^{A-1} \operatorname{Rect}_{L}[i-2 L(p+a P)-L / 2)\right],
$$

where $i=0,1, \cdots, N-1, p=0,1, \cdots, P-1$, and $A=\lfloor N /(2 P L)\rfloor$. From equation (5), one may realize that the angles of the elements of $\mathbf{R}$ relevant to user $p$ are a linear function of $\epsilon_{p}$. Hence, $\epsilon_{p}$ can be estimated as

$$
\epsilon_{p}=\frac{N}{A(A-1) L^{2}} \sum_{\substack{a=0 \\ a^{\prime}}}^{A-1} \sum_{\substack{\prime \\ a^{\prime} \neq a}}^{A-1} \sum_{i=0}^{L-1 L-1} \sum_{j=0} \frac{\angle R\left[i+i_{p, a}, j+i_{p, a^{\prime}}\right]}{2 \pi\left(i-j+2 L P\left(a-a^{\prime}\right)\right)}
$$

where $i_{p, a}=2 L(p+a P)$. It is worth mentioning that we calculate $\mathbf{R}$ once for all the users. Thus, the computational complexity with respect to the number of complex multiplication is $\mathcal{O}\left(M N^{2}\right)$, which increases linearly by increasing the number of BS antennas, $M$.

\section{CFO COMPENSATION}

Representing the convolution of channel and $\kappa^{\text {th }}$ OFDM symbol after CP removal as $\mathbf{H}_{m, p} \mathbf{x}_{p}^{\kappa}$, the received signal at the $m^{\text {th }} \mathrm{BS}$ antenna in the frequency domain can be written as

$$
\overline{\mathbf{r}}_{m}^{\kappa}=\sum_{p=0}^{P-1} \mathbf{F}_{N} \boldsymbol{\Phi}_{p}^{\kappa} \mathbf{H}_{m, p} \mathbf{x}_{p}^{\kappa}+\mathbf{F}_{N} \mathbf{n}_{m},
$$

where $\mathbf{H}_{m, p}$ is a circulant matrix with the first column $\mathbf{h}_{m, p}$ which is zero-padded to have the length of $N$. Note that we perform CFO compensation on a symbol by symbol basis to detect data. Thus, the superscript $\kappa$ can be dropped without any loss of generality. Considering $\mathbf{F}_{N}^{\mathrm{H}} \mathbf{F}_{N}=\mathbf{I}_{N}$, equation (8) can be expanded as

$$
\overline{\mathbf{r}}_{m}=\sum_{p=0}^{P-1} \mathbf{F}_{N} \boldsymbol{\Phi}_{p} \mathbf{F}_{N}^{\mathrm{H}} \mathbf{F}_{N} \mathbf{H}_{m, p} \mathbf{F}_{N}^{\mathrm{H}} \mathbf{d}_{p}+\overline{\mathbf{n}}_{m},
$$

where $\overline{\mathbf{n}}_{m}=\mathbf{F}_{N}^{\mathrm{H}} \mathbf{n}_{m}$ is the frequency domain noise vector. Let $\mathbf{E}_{p} \triangleq \mathbf{F}_{N} \boldsymbol{\Phi}_{p} \mathbf{F}_{N}^{\mathrm{H}}$ and $\widetilde{\mathbf{H}}_{m, p} \triangleq \mathbf{F}_{N} \mathbf{H}_{m, p} \mathbf{F}_{N}^{\mathrm{H}}$, thus, we have

$$
\overline{\mathbf{r}}_{m}=\sum_{p=0}^{P-1} \mathbf{E}_{p} \widetilde{\mathbf{H}}_{m, p} \mathbf{d}_{p}+\overline{\mathbf{n}}_{m}
$$

It is worth noting that due to the circulant property of $\mathbf{H}_{m, p}$, $\widetilde{\mathbf{H}}_{m, p}$ is a diagonal matrix, [21].

Using the maximum ratio combiner (MRC) equalization, the equalizer coefficient at subcarrier $k$ of user $p$ at the $m^{\text {th }}$ BS antenna can be obtained as

$$
z_{m, p}[k]=\frac{\bar{h}_{m, p}^{*}[k]}{\left\|\overline{\mathbf{h}}_{p}[k]\right\|^{2}},
$$

where $\overline{\mathbf{h}}_{m, p}$ contains the $N$-point DFT coefficients of $\mathbf{h}_{m, p}$ and $\overline{\mathbf{h}}_{p}[k]=\left[\bar{h}_{0, p}[k], \bar{h}_{1, p}[k], \cdots, \bar{h}_{M-1, p}[k]\right]^{\mathrm{T}}$. As a result, the channel is equalized over each subcarrier by applying the MRC to the frequency domain signal $\overline{\mathbf{r}}_{m}$. Hence, the signal of the user $q$ is given by

$$
\overline{\mathbf{y}}_{q}=\sum_{m=0}^{M-1} \mathbf{z}_{m, q} \sum_{p=0}^{P-1} \mathbf{E}_{p} \widetilde{\mathbf{H}}_{m, p} \mathbf{d}_{p}+\sum_{m=0}^{M-1} \mathbf{z}_{m, q} \overline{\mathbf{n}}_{m},
$$

where $\mathbf{Z}_{m, p}=\operatorname{diag}\left(\mathbf{z}_{m, p}\right)$, and $\mathbf{z}_{m, p}=\left[z_{m, p}[0], \ldots, z_{m, p}[N-\right.$ 1]] $]^{T}$. Defining an $N \times N$ matrix,

$$
\boldsymbol{\Omega}_{q, p}=\sum_{m=0}^{M-1} \mathbf{z}_{m, q} \mathbf{E}_{p} \widetilde{\mathbf{H}}_{m, p},
$$

the $q^{\text {th }}$ user's signal can be written as

$$
\overline{\mathbf{y}}_{q}=\sum_{p=0}^{P-1} \boldsymbol{\Omega}_{q, p} \mathbf{d}_{p}+\tilde{\mathbf{n}}
$$

where $\tilde{\mathbf{n}}=\sum_{m=0}^{M-1} \mathbf{Z}_{m, q} \overline{\mathbf{n}}_{m}$. In the absence of CFO, $\boldsymbol{\Omega}_{q, p}$ becomes a diagonal matrix. However, in the presence of CFO, the matrix $\boldsymbol{\Omega}_{q, p}$ looses its diagonal property and becomes a banded matrix whose off-diagonal elements are the intercarrier interference (ICI) coefficients.

From [22], we know that $\boldsymbol{\Omega}_{q, q}$ is a circulant matrix and can be written as $\boldsymbol{\Omega}_{q, q}=\mathbf{F}_{N} \mathbf{Q}_{q, q} \mathbf{F}_{N}^{\mathrm{H}}$, where $\mathbf{Q}_{q, q}$ is a diagonal matrix and its diagonal elements can be calculated as

$$
\operatorname{diag}\left(\mathbf{Q}_{q, q}\right)=\operatorname{diag}\left(\boldsymbol{\Phi}_{q}\right) \otimes \breve{\boldsymbol{\rho}}_{q},
$$

where $\breve{\boldsymbol{\rho}}_{q}[i]=\boldsymbol{\rho}_{q}[-i]$. Therefore, $\boldsymbol{\Omega}_{q, q}^{-1}=\mathbf{F}_{N} \mathbf{Q}_{q, q}^{-1} \mathbf{F}_{N}^{\mathrm{H}}$, and the $q^{\text {th }}$ user's transmit signal can be obtained as

$$
\hat{\mathbf{d}}_{q}=\boldsymbol{\Omega}_{q, q}^{-1} \overline{\mathbf{y}}_{q}=\mathbf{d}_{q}+\sum_{\substack{p=0 \\ p \neq q}}^{P-1} \widetilde{\boldsymbol{\Omega}}_{q, p} \mathbf{d}_{p}+\boldsymbol{\Omega}_{q, q}^{-1} \tilde{\mathbf{n}},
$$

where $\widetilde{\boldsymbol{\Omega}}_{q, p}=\boldsymbol{\Omega}_{q, q}^{-1} \boldsymbol{\Omega}_{q, p}$ is the MUI matrix between users $q$ and $p$. Note that since different users' channels are uncorrelated, the elements of $\widetilde{\boldsymbol{\Omega}}_{q, p}$ tend to zero as $M$ tends to infinity. Thus, MUI will be averaged out. 


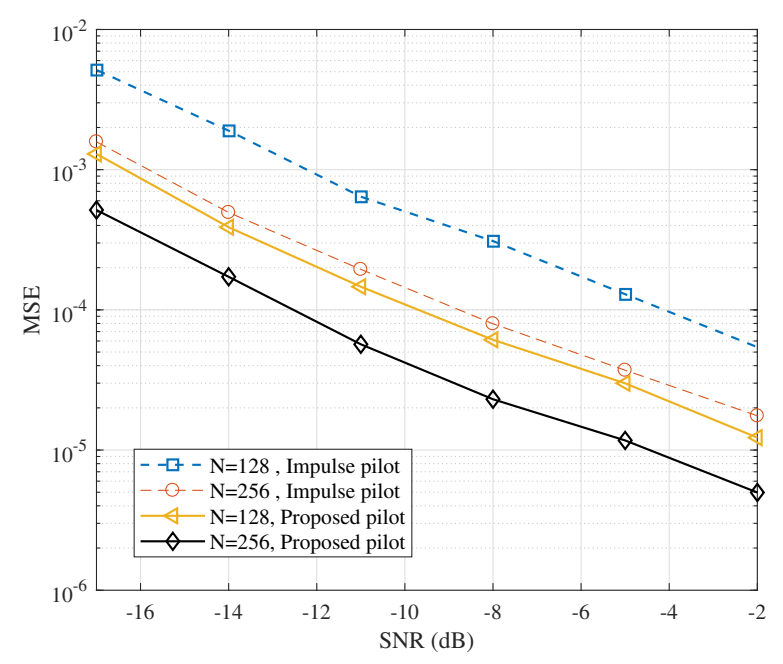

Fig. 1. MSE of the proposed CFO estimation technique for $M=200 \mathrm{BS}$ antennas, $P=4$ users and different number of subcarriers, $N$.

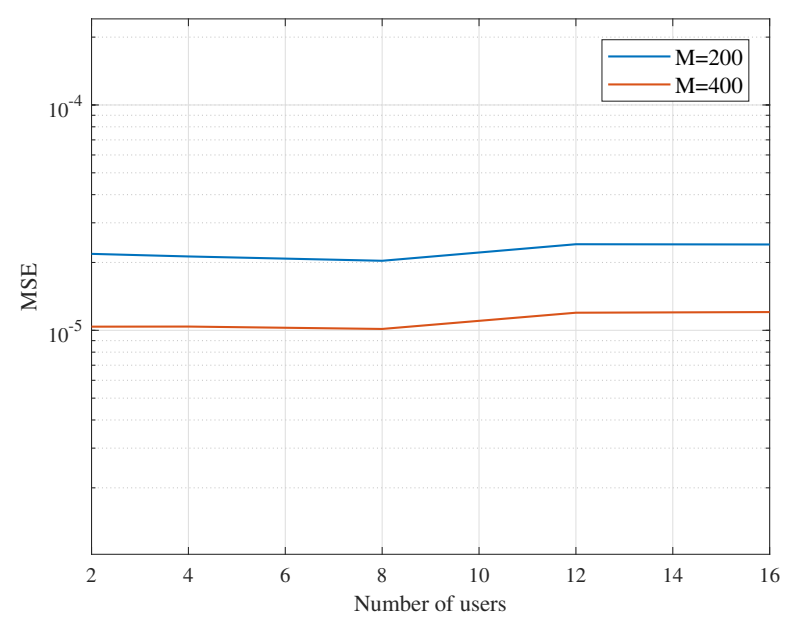

Fig. 2. MSE of the proposed CFO estimation technique with respect to the number of users, $P$, for $N=512$ subcarriers, and SNR $=-10 \mathrm{~dB}$.

\section{Numerical ANalysis}

In this section, we confirm our theoretical developments in the previous sections through numerical simulations. We assume that $P=4$ users are communicating with a BS with $M=200$ antennas. The extended typical urban (ETU) channel model, employed in Long Term Evolution (LTE) standard, is considered, [23], and the CP length is $N / 8$. The normalized $\mathrm{CFO}$ is randomly generated from a uniform distribution within the range $[-0.5,0.5)$. Fig. 1 presents the mean square error (MSE) of the proposed CFO estimation technique. The curves are compared to the case that the impulse-like pilots suggested for the single carrier transmission in [16] are employed. As it is shown, having the same pilot length for a fair comparison, we can achieve more accurate estimation by using the proposed pilot sequence, i.e. around $6 \mathrm{~dB}$ and $4 \mathrm{~dB}$ improvements

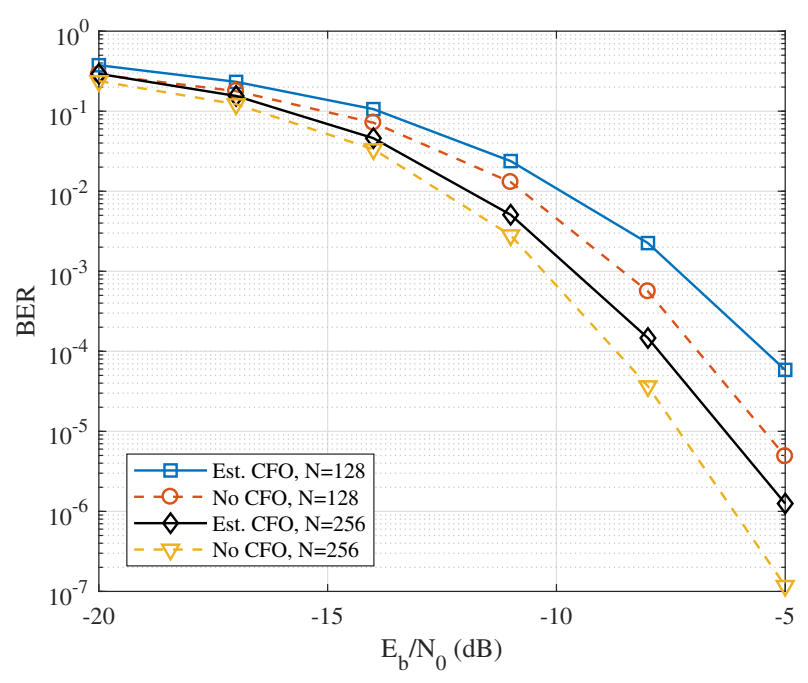

Fig. 3. BER performance of the proposed CFO estimation for $M=200 \mathrm{BS}$ antennas, $P=4$ users.

in MSE curves for $N=128$ and $N=256$ subcarriers, respectively. In fact, by using the impulse-like pilots, the covariance matrix of the received signals is a set of diagonal matrices. While by using the proposed rectangular-shaped pilots, the covariance matrix is a set of banded matrices, and consequently, we have a larger number of samples from which we can estimate the users' CFOs. In Fig. 2, it is depicted that the performance of our proposed technique is not affected by increasing the number of users. Moreover, the accuracy of the CFO estimation can be further enhanced by increasing the number of BS antennas. The bit error rate (BER) curves are depicted in Fig. 3 for $M=200$ BS antennas, $P=4$ users and different number of subcarriers. In the simulation, 4-QAM constellation is chosen, and MRC is used as a channel equalizer. The ML channel estimator is employed to estimate the channel between users and each BS antenna [24]. We have compared the BER performance of our proposed technique with that of the perfect synchronization as a benchmark.

\section{CONCLUSION}

In this paper, we proposed a CFO estimation technique for the uplink of OFDM-based multi-user massive MIMO systems. We have shown that if we calculate the covariance matrix of the received signal at the BS antennas, the users' CFOs can be estimated from the angles of the elements of this matrix. We have suggested real-valued pilot sequences that guarantee the orthogonality between users and also lead to an accurate estimation. Then, we have derived a closed form equation to estimate the users' CFOs. Thus, we do not need to perform any search algorithm like in the other existing CFO estimation techniques. Finally, the performance of our proposed $\mathrm{CFO}$ estimation technique was numerically evaluated, and we have also shown that the performance of our proposed technique does not deteriorate by increasing the number of users. 


\section{REFERENCES}

[1] J. G. Andrews, S. Buzzi, W. Choi, S. V. Hanly, A. Lozano, A. C. Soong, and J. C. Zhang, "What will 5G be?" IEEE Journal on Selected Areas in Communications, vol. 32, no. 6, pp. 1065-1082, 2014.

[2] E. G. Larsson, O. Edfors, F. Tufvesson, and T. L. Marzetta, "Massive MIMO for next generation wireless systems," IEEE Communications Magazine, vol. 52, no. 2, pp. 186-195, 2014.

[3] F. Rusek, D. Persson, B. K. Lau, E. G. Larsson, T. L. Marzetta, O. Edfors, and F. Tufvesson, "Scaling up MIMO: Opportunities and challenges with very large arrays," IEEE Signal Processing Magazine, vol. 30, no. 1, pp. 40-60, 2013.

[4] T. L. Marzetta, "Noncooperative cellular wireless with unlimited numbers of base station antennas," IEEE Transactions on Wireless Communications, vol. 9, no. 11, pp. 3590-3600, 2010.

[5] J. G. Andrews, T. Bai, M. N. Kulkarni, A. Alkhateeb, A. K. Gupta, and R. W. Heath, "Modeling and analyzing millimeter wave cellular systems," IEEE Transactions on Communications, vol. 65, no. 1, pp. 403-430, 2017.

[6] M. Ganji and H. Jafarkhani, "On the performance of MRC receiver with unknown timing mismatch-a large scale analysis," arXiv preprint arXiv:1703.10422, 2017.

[7] V. Kotzsch and G. Fettweis, "Interference analysis in time and frequency asynchronous network MIMO OFDM systems," in IEEE Wireless Communications and Networking Conference (WCNC), 2010, pp. 1-6.

[8] K. Raghunath and A. Chockalingam, "SIR analysis and interference cancellation in uplink OFDMA with large carrier frequency/timing offsets," IEEE Transactions on Wireless Communications, vol. 8, no. 5, 2009.

[9] T. M. Schmidl and D. C. Cox, "Robust frequency and timing synchronization for OFDM," IEEE transactions on communications, vol. 45, no. 12 , pp. 1613-1621, 1997.

[10] A. Farhang, N. Marchetti, L. E. Doyle, and B. Farhang-Boroujeny, "Low complexity CFO compensation in uplink OFDMA systems with receiver windowing." IEEE Transactions on Signal Processing, vol. 63, no. 10, pp. 2546-2558, 2015.

[11] H. Minn, V. K. Bhargava, and K. B. Letaief, "A robust timing and frequency synchronization for OFDM systems," IEEE Transactions on Wireless Communications, vol. 2, no. 4, pp. 822-839, 2003.

[12] V. Kotzsch, J. Holfeld, and G. Fettweis, "Joint detection and CFO compensation in asynchronous multi-user MIMO OFDM systems," in IEEE Vehicular Technology Conference (VTC), 2009, pp. 1-5.

[13] H. V. Cheng and E. G. Larsson, "Some fundamental limits on frequency synchronization in massive MIMO," in IEEE Asilomar Conference on Signals, Systems and Computers, 2013, pp. 1213-1217.

[14] H. Hojatian, M. J. Omidi, H. Saeedi-Sourck, and A. Farhang, "Joint CFO and channel estimation in OFDM-based massive MIMO systems," in IEEE International Symposium on Telecommunications (IST), 2016, pp. $343-348$.

[15] H. Huang, W. G. Wang, and J. He, "Phase noise and frequency offset compensation in high frequency MIMO-OFDM system," in IEEE International Conference on Communications (ICC), 2015, pp. 12801285.

[16] S. Mukherjee and S. K. Mohammed, "Low-complexity CFO estimation for multi-user massive MIMO systems," in IEEE Global Communications Conference (GLOBECOM), 2015, pp. 1-7.

[17] — , "Constant envelope pilot-based low-complexity CFO estimation in massive mu-mimo systems," in IEEE Global Communications Conference (GLOBECOM), 2016, pp. 1-5.

[18] — , "Information theoretic performance of periodogram-based CFO estimation in massive MU-MIMO systems," arXiv preprint arXiv:1611.04846, 2016.

[19] W. Zhang, F. Gao, and H.-M. Wang, "Frequency synchronization for massive MIMO multi-user uplink," in IEEE Global Communications Conference (GLOBECOM), 2016, pp. 1-6.

[20] W. Zhang, F. Gao, S. Jin, and H. Lin, "Frequency synchronization for uplink massive MIMO systems," IEEE Transactions on Wireless Communications, vol. 17, no. 1, pp. 235-249, 2018.

[21] G. Strang, G. Strang, G. Strang, and G. Strang, Introduction to linear algebra. Wellesley-Cambridge Press Wellesley, MA, 1993, vol. 3.

[22] P. Sabeti, A. Farhang, N. Marchetti, and L. Doyle, "Low-complexity CFO compensation for OFDM-based massive MIMO systems," in IEEE Globecom Workshops (GC Wkshps), 2017, pp. 1-7.
[23] W.-B. Yang, W.-B. Yang, and M. Souryal, LTE physical layer performance analysis. US Department of Commerce, National Institute of Standards and Technology, 2014.

[24] S. M. Kay, Fundamentals of statistical signal processing, volume I: estimation theory. Prentice Hall, 1993. 